1 Department of Respiratory Sciences, University of Leicester

2 Department of cardiovascular science, University of Leicester

3 UCL Medical School

4 Big Data Institute, University of Oxford

5 School of Medicine, University of Nottingham

Cite this as: BMJ 2021;375:n2709 http://dx.doi.org/10.1136/bmj.n2709 Published: 05 November 2021

\section{Covid-19 and ethnicity: we must seek to understand the drivers of higher transmission}

\author{
Daniel Pan, ${ }^{1}$ Shirley Sze, ${ }^{2}$ Christopher A Martin, ${ }^{1}$ Joshua Nazareth, ${ }^{1}$ Katherine Woolf, ${ }^{3}$ \\ Rebecca F Baggaley, ${ }^{1}$ T. Déirdre Hollingsworth, ${ }^{4}$ Kamlesh Khunti, ${ }^{2}$ Laura B Nellums, ${ }^{5}$ Manish Pareek
}

In early April 2020, as the first wave of the covid-19 pandemic hit the United Kingdom, concerns were raised regarding the disproportionately high numbers of people from ethnic minority groups being hospitalised and dying from covid-19. ${ }^{1}$ It was uncertain whether this was mainly due to a greater risk of acquiring the infection, greater risk of severe illness and death following infection-or both. By November 2020, a meta-analysis of over 18 million patients found a higher likelihood of infection in ethnic minority groups compared to White groups. ${ }^{2}$ This was confirmed by analysis of UK primary care data on the OpenSAFELY platform, which also found that the risk of testing positive between ethnic groups during the first wave was similar to the risk of covid-19 related death. ${ }^{3}$ Similarly, the REACT-2 study found that no differences existed in the infection-to-mortality ratio in ethnic minority groups compared to White groups despite high levels of SARS-CoV-2 antibodies in the ethnic minority population. ${ }^{4}$

It is increasingly recognised that the majority of disproportionate clinical outcomes from covid-19 is driven by public health factors, such as socioeconomic differences, occupation, and systemic exposure gaps rather than any genetic predisposition to severe illness. ${ }^{5}$ However, despite the major interest in disentangling the relationship between ethnicity and covid-19, surprisingly little attention has been given to examining the risk factors for infection, which unlike most of the risk factors for severe covid-19, are malleable and potentially reversible. This will become increasingly important as a higher proportion of patients in the community become vaccinated and countries start opening up economically.

Ethnicity is a complex phenomenon, with ethnic groups reflecting diverse aspects of culture, ancestry, language, and religion. Of particular relevance to the risk of SARS-CoV-2 infection are differences by ethnic group in experiences of structural discrimination, household structures (including multi-generational and multi-occupancy living) and health-related behaviours, and attitudes to vaccines.

Although large strides have been made in the last year to ensure ethnicity is included as a standard variable in a variety of different studies, the "ethnicity" variables used are broad (for example Asian, Black, White and Other) and studies do not adjust for factors such as those listed above, which may underpin the association between ethnicity and risk of SARS-CoV-2 infection. This lack of granularity makes it difficult to develop and test hypotheses about the causes of increased infection in different ethnic groups. In addition, interventions aiming to reduce SARS-CoV-2 transmission such as lockdown, mass testing, contact tracing and vaccination, have adopted a "one size fits all" approach, with limited consideration of the specific cultural, social, or language barriers that exist for ethnic minority groups, and an absence of auditing which interventions work, and which do not work, at local and regional levels.

With the policy of releasing all UK covid restrictions allowing for the rise of another wave of infection, there must now be a focused effort to research SARS-CoV-2 transmission among ethnic minority populations, aiming specifically to disentangle which factors are mediating the increased infection risk among specific ethnic minority groups compared to those of White British ethnicity. This should be supported by meaningful engagement with stakeholders and community influencers to maximise awareness and inform research, policy, and practice. The prevalence, by ethnic group, of novel variants of concern which are biologically more transmissible should also be monitored, since they may spread faster among ethnic minority communities.

Only by a deeper understanding of factors relating to disproportionate SARS-CoV-2 infections in ethnic minority groups compared to White groups can we act to reduce hospitalisation, intensive care unit admission, and death. A holistic approach is required to target other factors that may be important determinants of vaccine uptake that will often overlap with ethnic groups. Mathematical models calculating the reproductive number $(R o)$ of new variants, and based on data from other ethnic groups, should also take into account any overestimations of transmission if the variants themselves were introduced by people from multigenerational households. In these circumstances, increased viral transmission is not due to any altered biological properties of the virus, but by the increased living densities commonly associated with ethnic minority households.

Finally, we call for an increased effort to include ethnic minority groups in covid-19 clinical trials. A report by the National Institute for Health Research found that ethnic minority groups comprise $9.3 \%$ of participants in covid-19 studies and $5.7 \%$ in vaccines studies. These figures are well below their representation in the national population $(13.8 \%){ }^{6}$ This underrepresentation, along with continued vaccine hesitancy and lower treatment uptake all suggests that ethnic minority groups, including healthcare workers, all continue to lack trust in Western medical healthcare systems. ${ }^{7}$ Additional access factors specific to ethnic minority groups, such 
as difficulties taking time off work and language barriers may also be responsible.

Over the 22 months of the pandemic, we have learnt that ethnic minority groups in Western countries are disproportionately affected by covid-19 primarily because of higher transmission. Now that SARS-CoV-2 is here to stay, we must seek to understand the drivers of higher transmission in these vulnerable groups and make concerted efforts to include them in interventions, to minimize ethnic minority groups being yet again disproportionately affected by covid-19 in the waves ahead.

\section{Competing interests: none declared}

Provence and peer review: not commissioned, not peer reviewed

1 Pareek M, Bangash MN, Pareek N, etal. Ethnicity and COVID-19: an urgent public health research priority. Lancet 2020;395:1421-2. doi: 10.1016/S0140-6736(20)30922-3 pmid: 32330427

2 Sze S, Pan D, Nevill CR, etal. Ethnicity and clinical outcomes in COVID-19: A systematic review and meta-analysis. EClinicalMedicine 2020;29:100630. doi: 10.1016/j.eclinm.2020.100630. pmid: 33200120

3 Mathur R, Rentsch CT, Morton CE, etalOpenSAFELY Collaborative. Ethnic differences in SARS-CoV-2 infection and COVID-19-related hospitalisation, intensive care unit admission, and death in 17 million adults in England: an observational cohort study using the OpenSAFELY platform. Lancet 2021;397:1711-24. doi: 10.1016/S0140-6736(21)00634-6 pmid: 33939953

4 Ward $\mathrm{H}$, Atchison C, Whitaker M, etal. Antibody prevalence for SARS-CoV-2 following the peak of the pandemic in England: REACT2 study in 100000 adults.medRxiv 2020; 2020.08.12.20173690doi: 10.1101/2020.08.12.20173690.

5 Learoyd AE, Douiri A, Hart N. COVID-19 and ethnicity: has history repeated itself? Thorax 2021;76:537-8. doi: 10.1136/thoraxjnl-2021-216992 pmid: 33837142

6 National Institute for Health Research. NIHR research ethnicity data provides insight on participation in COVID-19 studies. 2020.

https://www.nihr.acuk/news/nihr-research-ethniaty-data-provides-insight-on-partiapation-in-covid-19-studies/26460.

7 Woolf K, McManus IC, Martin CA, etal. Ethnic differences in SARS-CoV-2 vaccine hesitancy in United Kingdom healthcare workers: Results from the UK-REACH prospective nationwide cohort study.medRxiv 2021; 000: 2021.04.26.21255788. 